\title{
ENDOSCOPIA DE CONTATO(MICROESTOMATOSCOPIA) NAS LESÕES DA BOCA E DO LÁBIO: AVALIAÇÃO DO MÉTODO
}

\section{CONTACT ENDOSCOPY (MICROSTOMATOSCOPY) IN ORAL LESIONS: EVALUATION OF THE METHOD}

\author{
Gyl H A Ramos, TCBC-PR'; Marcos R Tavares, TCBC-SP²; Rogério A Dedivitis, TCBC-SP3; \\ Cristiane M. França ${ }^{4}$; Benedito V. Oliveira ${ }^{5}$; Paola A Pedruzzi ${ }^{6}$
}

\begin{abstract}
RESUMO: Objetivo: Descrever as dificuldades da endoscopia de contato aplicada à boca e ao lábio (microestomatoscopia de contato) e aplicar nas lesões do lábio os critérios usados na endoscopia de contato de outras topografias. Método: Estudo prospectivo utilizando o endoscópio de contato Storz 8715A e o corante azul de toluidina. Vinte exames foram feitos para aprendizado da técnica e depois mais 31 (21 lesões do lábio e 10 da boca), todos exames in vivo. Os critérios avaliados foram: Celularidade, Arquitetura, Estratificação e Morfologia. Resultados: A falta de nitidez, os tremores finos e o deslizamento do aparelho dificultaram o procedimento em $100 \%$ dos casos, mas permitiram um exame adequado. Os 13 diagnósticos de malignidade das lesões do lábio apresentaram todos os critérios alterados. Conclusão: as dificuldades encontradas foram o contato do aparelho com a superfície anatômica, o deslizamento do aparelho, os tremores finos e a falta de nitidez da imagem, que não inviabilizaram a realização e a interpretação do exame. Os critérios são aplicáveis às lesões da boca e do lábio inferior (Rev. Col. Bras. Cir. 2008; 35(6): 355-360).
\end{abstract}

Descritores: Neoplasias bucais; Neoplasias labiais; Carcinoma; Endoscopia/métodos; Diagnóstico precoce.

\section{INTRODUÇÃO}

O câncer da cavidade oral está dentre os 10 mais comuns cânceres no mundo e acomete principalmente os homens ${ }^{1}$. A estimativa de novos casos e mortes por câncer bucal em 2007 nos EUA foi de 22.560 e 5.370, respectivamente ${ }^{2}$. No Brasila taxa de incidência do câncer da cavidade oral estimada é de $14.160^{3}$ Cerca de 50\% desses tumores são diagnosticados tardiamente (estádio III ou IV) ${ }^{4}$.

A citologia exfoliativa pode auxiliar no diagnóstico, assim como o azul de toluidina pode orientar a biópsia de lesões duvidosas ao exame clínico ${ }^{5,6}$. Esses métodos não têm aplicação clínica totalmente segura devido aos índices de falso-positivos ou falso-negativos apresentados. Portanto, existe a necessidade de encontrar-se um método não invasivo e confiável para o diagnóstico precoce das lesões malignas, in vivo ${ }^{7}$.

O colposcópio com aumento óptico de até 40 vezes foi aplicado para o auxílio diagnóstico de lesões da cavidade bucal $^{8}$, com resultados satisfatórios, principalmente se associado ao teste do azul de toluidina (coloração utilizada na microestomatoscopia $)^{9,10 .}$
Outro instrumento utilizado pela ginecologia e adaptado para o uso na cavidade bucal é o microcolpohisteroscópio, que foi patenteado por Hamou em 1979 para exame do epitélio do colo uterino, com capacidade de aumentos de 60 e 150 vezes $^{11,12}$.

O método foi aplicado em outras topografias ${ }^{13-18}$, inclusive na mucosa da cavidade bucal. L'Estrange, em $1989^{19}$, citou que esse método poderia ser a ponte entre a microscopia de superfície da mucosa e a histologia. Salientou que outros estudos seriam necessários para estabelecer o grau de correlação existente entre a endoscopia de contato e a histologia. A partir de 2004 outras pesquisas relacionadas à cavidade bucal foram apresentadas à comunidade médica, voltadas para lesões benignas ${ }^{20} \mathrm{e} / \mathrm{ou}$ malignas ${ }^{21}$.

Todos os estudos baseiam-se nas alterações já conhecidas pela citopatologia, como: as nucleares e o conseqüente aumento da relação núcleo-citoplasmática; a maior densidade celular por campo; mitoses atípicas ou normais na camada superficial; as mudanças do padrão de distribuição da camada superficial do epitélio; a perda da maturação celular nessa camada, entre outras ${ }^{22-26}$. Independentemente do método diagnóstico utilizado, um resultado positivo deve ser confirmado por biópsia.

1. Mestre pelo Curso de Pós Graduação em Cirurgia de Cabeça e Pescoço do Hospital Heliópolis; Doutorando pelo Serviço de Cirurgia de Cabeça e Pescoço da Faculdade de Medicina da Universidade de São Paulo (USP); Cancerologista, Titular do Serviço de Cirurgia de Cabeça e Pescoço do Hospital Erasto Gaertner (HEG) em Curitiba.

2. Cirurgião de Cabeça e Pescoço; Mestre e Doutor em Medicina; Professor Livre Docente do Serviço de Cirurgia de Cabeça e Pescoço da Faculdade de Medicina da Universidade de São Paulo (SP).

3. Doutor em Medicina pela Escola Paulista de Medicina, Serviço de Cirurgia de Cabeça e Pescoço do hospital Ana Costa, Santos.

4. Doutora em Odontologia (Patologia Bucal) pela Universidade de São Paulo (USP), Titular da Disciplina de Patologia Geral e Bucal da Universidade Metropolitana de São Paulo.

5. Cancerologista e Cirurgião de Cabeça e Pescoço; Chefe do Serviço de Cirurgia de Cabeça e Pescoço do Hospital Erasto Gaertner (HEG).

6. Mestre; Cancerologista; Titular do Serviço de Cirurgia de Cabeça e Pescoço do Hospital Erasto Gaertner (HEG).

Recebido em 16/05/2008

Aceito para publicação em 28/08/2008

Conflito de interesses: nenhum

Fonte de financiamento: nenhuma

Trabalho realizado no Serviço de Cirurgia de Cabeça e Pescoço do Hospital Erasto Gaertner. 
Os objetivos deste estudo são de descrever as dificuldades deste exame (endoscopia de contato ou microestomatoscopia) quando aplicados às lesões da boca e do lábio inferior (inclundo o vermelhão), bem como verificar se os critérios já usados neste mesmo tipo de exame para outras topografias são também passíveis de análise nestes locais.

\section{MÉTODO}

O estudo foi prospectivo, de casos não consecutivos de lesões do lábio inferior e da cavidade bucal, e foi desenvolvido no período compreendido entre dois de dezembro de 2004 e 28 de fevereiro de 2005, interessando a endoscopia de contato de lesões in vivo, no ambulatório.

O projeto foi aprovado pela Comissão de Ética e Pesquisa do Hospital Erasto Gaertner sob a identificação pp 1149 (em cinco de abril de 2005, com validade retrospectiva).

Foram incluídos os pacientes:

A) Primeiros 20 casos: 1- Do Ambulatório de Cirurgia de Cabeça e do Pescoço do Hospital Erasto Gaertner em Curitiba, provenientes do atendimento habitual desse hospital; do Ambulatório de Cirurgia da Cabeça e do Pescoço do Departamento de Otorrinolaringologia da Universidade Federal do Paraná; do Centro de Diagnóstico do Sistema de Saúde Municipal (nível secundário); da clínica privada do pesquisador; 2- Com ou sem lesão das mucosas da boca ou do lábio inferior, inclusive do vermelhão; 3- Com ou sem biópsia; 4Sem exposição prévia regional à radioterapia; 5- Sem tratamento cirúrgico sobre o sítio da lesão; 6- Sem tratamento quimioterápico prévio.

B) Após o 20 caso: 1- Do Ambulatório de Cirurgia de Cabeça e do Pescoço do Hospital Erasto Gaertner em Curitiba, provenientes do atendimento ambulatorial habitual desse hospital e da clínica privada do pesquisador 2Com lesão das mucosas da boca ou do lábio inferior, inclusive do vermelhão; 3- Com ou sem biópsia, sendo que a suspeição clínica de malignidade foi comprovada por biópsia; 4- Sem exposição prévia à radioterapia; 5- Sem tratamento cirúrgico sobre o sítio da lesão; 6- Sem tratamento quimioterápico prévio.

A) Primeiros 20 casos: Em relação à topografia da lesão examinada, cinco foram do lábio inferior e 15 da cavidade bucal, todos in vivo e corados com azul de toluidina. $\mathrm{O}$ aspecto clínico macroscópico foi de candidíase em um caso, eritroplasia em três, leucoplasia em 10, ulceração maligna em quatro e vasos atípicos em um e normal em outro.
B) Após o $2{ }^{\circ}$ caso: Foram incluídos 31 pacientes, 10 com lesão na boca (32\%) e 21 no lábio (68\%). Das lesões da cavidade bucal, quatro foram na língua, três no assoalho, uma no palato duro, uma na área retromolar e uma na mucosa jugal.

Os aspectos clínicos das lesões do lábio e da boca estão descritos na tabela 1 . O resumo da casuística após o $20^{\circ}$ caso está no tabela 2.

As primeiros 20 microestomatoscopias de contato foram feitas com a finalidade de adquirir os conhecimentos práticos básicos necessários para a continuação do estudo e os demais foram utilizados para atingir os objetivos propostos.

Todos os pacientes receberam esclarecimentos quanto ao estudo e ao exame, que foi agendado após terem concordado por escrito. Os exames foram in vivo, isto é, realizados diretamente no indivíduo, ambulatorialmente. A posição dos pacientes, deitados ou sentados, foi escolhida de acordo com as necessidades de conforto deles, e do posicionamento e apoio do aparelho. Não foi utilizado qualquer tipo de anestesia, a não ser quando feita a biópsia.

Os exames e as respectivas análises das imagens foram feitos pelo mesmo profissional médico, especialista em Cirurgia de Cabeça e Pescoço, com assessoria de patologistas.

A) Equipamento: O aparelho utilizado para este estudo foi o laringoscópio de contato, modelo 8715A da Karl Storz, com angulação zero grau, 4 mm de diâmetro, que proporciona aumentos da imagem de 60 e 150 vezes. A microcâmera foi a Karl Storz 202321 - 20, a fonte de luz foi a Karl Storz Xenon 175, o videocassete foi Sony SLV 40 $\mathrm{BR}$, a digitalização das imagens VHS foi feita pelo aparelho Sansung DVD Recorder DVDR 150, o monitor de TV foi Sony KV 1450 B e o computador utilizado foi o Toshiba A 55 Series.

B) Técnica utilizada: $O$ exame de endoscopia de contato (microestomatoscopia de contato) consistiu no contato

Tabela 1 - Aspecto clínico das lesões da boca e do lábio.

\begin{tabular}{lccc}
\hline Aspecto Clínico & Lábio & Boca & Total \\
\hline Leucoplasia & 4 & 5 & 9 \\
Carcinoma & 6 & 2 & 8 \\
Ceratose & 6 & 0 & 6 \\
Normal ou hiperemia & 3 & 3 & 6 \\
Vegetação e crostas & 1 & 0 & 1 \\
Submucoso & 1 & 0 & 1 \\
Total & 21 & 10 & 31 \\
\hline
\end{tabular}

Tabela 2 - Resumo da casuística.

\begin{tabular}{|c|c|c|c|}
\hline Casuistica / Local & Primeiros 20 Exames & Do $21^{\circ}$ Em Diante & Total \\
\hline Lábio & 5 & 21 & 26 \\
\hline Boca & 15 & 10 & 25 \\
\hline Total & 20 & 31 & 51 \\
\hline
\end{tabular}


da lente do endoscópio com a mucosa, com o vermelhão e/ou com a lesão. As imagens obtidas foram gravadas pelo sistema de vídeo VHS, convertidas para DVD (digitalizadas) e instaladas no computador. Desses arquivos foram gravadas fotos para documentação e análise posterior.

A rotina da coloração dos locais a serem examinados foi: 1- Higiene local com soro fisiológico, aplicado com gaze umedecida; 2 - Aplicação de solução aquosa de ácido acético a $1 \%$ por um minuto, para facilitar a remoção da saliva e o excesso de fibrina; 3 - Aplicação de solução aquosa de azul de toluidina a $1 \%$, por um minuto; 4 - Retirada do excesso de corante com gaze embebida em soro fisiológico.

\section{C) Método específico para após o vigésimo caso:}

$\mathrm{O}$ exame foi de toda a lesão e da sua periferia. As dificuldades e soluções encontradas foram anotadas para posterior análise, assim como as alterações conforme os critérios utilizados. Quando realizada a biópsia teve o local definido pelo aspecto macroscópico e, portanto, não foi direcionada pela microestomatoscopia de contato.

Foram coletados dados referentes às dificuldades e limitações técnicas relacionadas ao aparelho (laringoscópio de contato) e às características anatômicas da boca e do lábio. Foi utilizada a seguinte classificação: sem dificuldades, pouca dificuldade (permite o exame em mais de $90 \%$ dos casos) e muita dificuldade (permite o exame em menos de $50 \%$ dos casos).

A qualidade da imagem foi classificada como suficiente ou insuficiente, considerando a capacidade da geração, da captura e da visualização de núcleos e/ou da superfície do vermelhão.

Aspectos histológicos / citológicos: Interessaram a celularidade, que é a quantidade de núcleos visualizados; a arquitetura, que é a forma da distribuição das células; e a estratificação, que é a existência de células com maturação compatível com a camada superficial: a morfometria, que são as características do núcleo e sua relação com o citoplasma. São detalhados a seguir.

Celularidade: classificada como normal ou aumentada, usando como referência os achados da microestomatoscopia de contato de áreas sem lesão da mucosa bucal, labial, e ou da superfície do vermelhão dos mesmos pacientes, sob aumento de 150 x. Arquitetura: normal ou alterada, considerando o arranjo das células da camada superficial à microestomatoscopia de contato, sob os aumentos de 60 e 150 vezes. Foi usada a mesma referência do critério Celularidade. Estratificação: normal ou alterada, conforme encontradas células compatíveis ou não com o esperado para a camada superficial do vermelhão ou para a camada superficial da mucosa da boca. Morfometria: normal ou alterada, conforme encontrado: alteração da forma do núcleo; acentuada concentração do corante no mesmo; aumento da relação núcleo-citoplasma; presença de nucléolo hipercromático; presença de figuras mitóticas (Figura 1).

D) Análise estatística: As variáveis foram analisadas pelo estatístico Dinarte Orlandi (CONRE n ${ }^{\circ}$ 7520-A), da Liga Paranaense de Combate ao Câncer, utilizando análise descritiva, análise de regressão e o teste de correlação de
Kendall, pelo software estatístico Statistical Package for Social Sciences (SPSS) versão 12.0. O nível de significância considerado foi de $5 \%$.

\section{RESULTADOS}

As dificuldades encontradas em relação ao aparelho e às estruturas anatômicas examinadas (lábio e cavidade bucal) foram referentes ao contato da superfície da lente, aos tremores finos e ao deslizamento do aparelho. Essas dificuldades variaram conforme a topografia, o que está exposto na tabela 3. A média de tempo para cada exame, sem a realização da biópsia, foi de 12 minutos.

Em todos os casos a nitidez da imagem obtida não foi perfeita, mas foi possível a identificação das estruturas e sua avaliação. Esta nitidez variou conforme o sítio da lesão, a quantidade de ulceração, o volume de crostas, a eficiência da limpeza prévia do local, a colaboração do paciente, a presença de saliva, a mobilidade da estrutura e o apoio para o aparelho (Tabela 4).

Os critérios aplicados às 21 microestomatoscopias de contato das lesões do lábio incluídos após o vigésimo caso (Celularidade, Arquitetura, Estratificação e Morfometria), seus resultados e suas combinações correlacionadas com o diagnóstico da microestomatoscopia de contato estão na tabela 5. A combinação no diagnóstico de malignidade foi: Celularidade aumentada, Estratificação alterada, Arquitetura alterada e Morfometria altera, em 100\% dos casos.

\section{DISCUSSÃO}

Por ser um procedimento agressivo, a biópsia não é facilmente aceita pelas pessoas, principalmente quando a le-

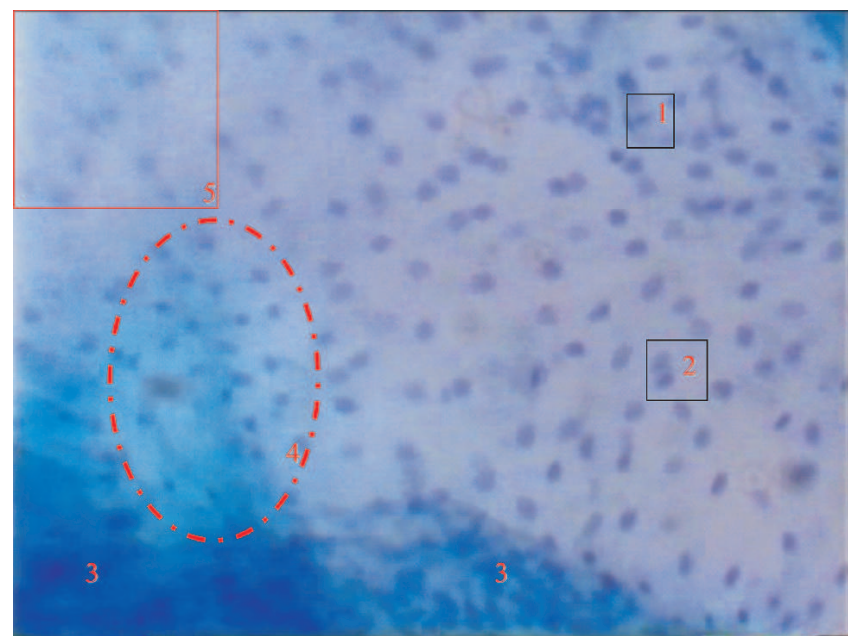

Figura 1 - Microestomatoscopia de lesão do dorso da língua, sob aumento de $150 x$

Os principais itens (exceto vasos) a serem analisados são evidenciados: 1 - célula em metáfase; 2 - células em citocinese; 3 -área tumoral, com provável queratina, difícil discernir citoplasma elou núcleo. Maior celularidade; 4 - núcleos com área menor, pode ser atipia; 5 borramento provavelmente por estiramento do tecido na hora do contato com o aparelho.

Fonte: Ramos et al. $(2006)^{26}$. 
Tabela 3 - Local examinado, dificuldades encontradas e sua influência na realização do exame (após o $20^{\circ}$ caso).

\begin{tabular}{llll}
\hline $\begin{array}{l}\text { Topografia } \\
\text { (Número de casos) }\end{array}$ & Contato*(Lente X Lesão) & Tremores Finos** & Deslizamento do Aparelho*** \\
\hline Lábio (21) & Não & Muito & Pouco \\
Soalho bucal (3) & Pouca & Pouco & Pouco \\
Língua (4) & Pouca & Pouco & Pouco \\
Palato duro (1) & Muita & Muito & Pouco \\
Jugal (1) & Não & Pouco & Muito \\
Retromolar (1) & Pouca & Pouco & Pouco \\
\hline
\end{tabular}

Legenda: * Sem dificuldades; pouca (permite exame em mais de $90 \%$ dos casos); muita (permite exame em menos de $50 \%$ dos casos). ***, ** pouco (atrapalha em menos de $50 \%$ dos casos) e muito (atrapalha a coleta de imagens em mais de $90 \%$ dos casos).

Tabela 4 - Fatores que influenciaram a qualidade da imagem.

\author{
Sítio da Lesão \\ Ulceração \\ Volume de Crostas \\ Limpeza \\ Quantidade de Saliva \\ Mobilidade da Estrutura \\ Apoio para o Aparelho \\ Colaboração do Paciente
}

são é assintomática e, mais ainda, se é proposta em campanhas de saúde bucal ${ }^{27}$.

O azul de toluidina é um corante metacromático do grupo das tiazinas, solúvel em água e em álcool ${ }^{28}$, tem afinidade e provoca coloração evidente tanto no meio intranuclear quanto citoplasmático. Promove uma visualização do número de núcleos e de mitoses melhor do que o corante azul de metileno ${ }^{29}$.

O aparelho utilizado para este estudo foi o mesmo da endoscopia de contato da laringe, uma óptica com angulação zero grau e $4 \mathrm{~mm}$ de diâmetro. Esse comprimento acentua os finos tremores das mãos, provocando a piora da nitidez da imagem, principalmente sob o aumento de 150 vezes. Os tre- mores estão presentes em mais de $90 \%$ dos exames do lábio e do palato duro.

A necessidade de ter um apoio para o aparelho foi suprida com a adaptação de um suporte para braço, usado para a coleta de sangue nos laboratórios. Já para os tremores finos das mãos o uso do rinoscópio de contato, com $16 \mathrm{~cm} \mathrm{de}$ comprimento e $4 \mathrm{~mm}$ de diâmetro pode ser a solução.

A angulação de zero grau implicou dificuldades de contato principalmente no palato duro e na área retromolar, sendo que a utilização de óptica com angulação de 30 graus pode minimizar o problema.

O deslizamento do aparelho em decorrência da saliva foi mais frequiente na região jugal, seguido do ventre lingual. Uma secagem prévia com gaze e a respiração bucal provocando o ressecamento do local, facilitaram o procedimento.

Todas estas particularidades ou dificuldades não impossibilitaram o exame, pois a captura e a qualidade das imagens foram suficientes para a análise dos critérios utilizados. Esta qualidade dependeu: do sítio da lesão; da quantidade de ulceração; do volume de crostas; da limpeza prévia do local; da colaboração do paciente; da presença de maior ou menor quantidade de saliva; da mobilidade da estrutura examinada; e do apoio para o aparelho.

Os critérios aplicados foram escolhidos por serem já consagrados pela citopatologia ${ }^{30} \mathrm{e}$ pela histopatologia ${ }^{26}$ além do fato de terem sido usados e citados por estudos anteriores de endoscopia de contato ${ }^{10,18,23-25}$.

Tabela 5 - Descrição do número de casos, resultado dos Critérios usados à microestomatoscopia de contato e as combimações encontradas, conforme o diagnóstico nas lesões do lábio inferior.

\begin{tabular}{llllll}
\hline $\mathbf{N}^{\mathbf{0}}$ & Celularidade & Estratificação & Arquitetura & Morfometria & Diagn EC \\
\hline 13 & Aumentada & Alterada & Alterada & Alterada & Maligno \\
1 & Aumentada & Alterada & Alterada & Normal & Benigno \\
1 & Aumentada & Normal & Alterada & Alterada & Benigno \\
1 & Aumentada & Normal & Alterada & Alterada & Suspeito \\
2 & Aumentada & Normal & Normal & Normal & Benigno \\
3 & Normal & Normal & Normal & Normal & Benigno \\
\hline
\end{tabular}

Diagn Ec = Diagnóstico da endoscopia de contato.

Fonte: Pesquisador. 
A capacidade de identificar estes critérios e suas alterações, nas condições deste exame neste estudo, foi comprovada pela combinação encontrada com base nos diagnósticos da endoscopia de contato e do respectivo anatomopatológico. A combinação mais comum para o diagnóstico de malignidade foi Celularidade aumentada, Estratificação alterada, Arquitetura alterada e Morfometria alterada (tabelas 3). Por ser uma combinação coerente, confiouse na utilidade e na reprodutibilidade dos critérios da endoscopia de contato nas lesões da cavidade bucal e do lábio (microestomatoscopia).

As dificuldades encontradas no estudo Inicial foram a falta de nitidez, os tremores finos e o deslizamento do apare- lho em todos os exames e a falta de contato do aparelho com a superfície mucosa de algumas das regiões da boca. No entanto não inviabilizaram o exame.

Os critérios Celularidade, Arquitetura, Estratificação e Morfometria, testados no Estudo Inicial e aplicados ao lábio inferior, foram passíveis de identificação e análise.

\section{AGRADECIMENTOS}

Ao dentista Shozo Miyachi, pelo encaminhamento de pacientes dos primeiros 20 exames. A Patrícia Varella pelo auxílio na formatação para a revista. Ao Hospital Erasto Gaertner.

\begin{abstract}
Background: 1- To describe difficulties of contact endoscopy related to mouth and lip (contact microstomatoscopy) and 2-apply the criteria used in contact endoscopy of other topographies when lip lesions are diagnosed. Methods: A prospective study was performed with the contact endoscope Storz 8715 A and toluidine blue was used. Twenty examinations were accomplished in order to learn the technique; after that, other 31 were included (21 lip lesions and 10 oral lesions), all in vivo examinations. The criteria assessed were: cellularity, architecture, stratification and morphology. Results: Lack of image clearness, slight tremors and sliding of the equipment made the procedure difficult in 100\% of the cases; even though, accomplishment of proper examination was obtained. The 13 cases of lip malignancy lesions had the criteria altered as a whole. Conclusion: Difficulties found were the contact of the equipment with anatomic surface, the sliding of the equipment, the slight tremors, and the lack of image clearness. Nevertheless, such elements did not interrupt the accomplishment and the examination analysis. The criteria are applied to oral and lower lip lesions.
\end{abstract}

Key words: Mouth neoplasm; Lip neoplasm; Carcinoma; Endoscopy/methods; Early diagnosis.

\section{REFERÊNCIAS}

1. Scully C, Newman L, Bagan JV. The role of the dental team in preventing and diagnosing cancer: oral cancer diagnosis and screening. Dent Update. 2005; 32(6):326-8, 331-2, 335-7.

2. National Cancer Institute. Oral cancer [cited dez 2007]. Available from: www.cancer.gov/ cancertopics/ types/oral

3. Brasil. Ministério da Saúde. Secretaria de Assistência à Saúde. Instituto Nacional do Câncer, INCa. Estimativa 2008. Disponível em: http://www.inca.gov.br/ estimativa/2008. Acessado em dez. 2007.

4. Holmes JD, Dierks EJ, Homer LD, Potter BE. Is detection of oral and oropharyngeal squamous cancer by a dental health care provider associated with a lower stage at diagnosis? J Oral Maxillofac Surg. 2003; 61(3):285-91.

5. Mashberg A, Samit A. Early diagnosis of asymptomatic oral and oropharyngeal squamous cancers. CA Cancer J Clin. 1995; 45(6):328-51.

6. Silverman S Jr. Early diagnosis of oral cancer. Cancer. 1988; 62(8 Suppl):1796-9.

7. Olivo M. Endoscopic fluorescence imaging to detect neoplasia in oral cavities. Cancer update (National Cancer Centre Singapore). 2004, v.3.

8. Shedd DP, Hukill PB, Bahn S. In vivo staining properties of oral cancer. Am J Surg. 1965;110(4):631-4.
9. Oliveira BV, Ramos GHA, Sampaio LA, Biasi L. Uso do colposcópio na avaliação de lesões da boca. Rev Bras Cir Cabeça Pescoço. 2007, 36:83-6.

10. Gynther GW, Rozell B, Heimdahl A. Direct oral microscopy and its value in diagnosing mucosal lesions: a pilot study. Oral Surg Oral Med Oral Pathol Oral Radiol Endod. 2000; 90(2):164-70.

11. Hamou JE. Microhysteroscopy. Clin Obstet Gynecol. 1983;26(2):285-301.

12. Hamou JE. Microendoscopy and contact endoscopy. Brevet Français 79; 04168 Paris, 1979; International Patent PCT/FR80/ 0024, Paris, 1980.US Patent 4.385.810. Washington, DC, 1983.

13. Andrea M, Dias O. Rigid and contact endoscopy associated to microlaryngeal surgery. Arq Portugueses ORL. Pat Cerv-Fac, Lisboa [Suppl2], 1994.

14. Andrea M, Dias O, Macor C, Santos A, Varandas J. Contact endoscopy of the nasal mucosa. Acta Otolaryngol. 1997; 117(2):307-11

15. Xiaoming H, Haiqiang M, Manquan D, Jianyong S, Kela L, Xiaoman L, Tengbo H. Examination of nasopharyngeal epithelium with contact endoscopy. Acta Otolaryngol. 2001;121(1):98-102.

16. Wai Pak M, To KF, Lee JC, Liang EY, van Hasselt CA. In vivo real-time diagnosis of nasopharyngeal carcinoma using contact rhinoscopy. Head Neck. 2005; 27(11):1008-13.

17. Romano FR, Voegels RL, Goto EY, Prado FA, Butugan O. Nasal contact endoscopy for the in vivo diagnosis of inverted 
schneiderian papilloma and unilateral inflammatory nasal polyps. Am J Rhinol. 2007; 21(2):137-44.

18. Dedivitis RA, Guimarães AV. Contact endoscopy for intraoperative parathyroid identification. Ann Otol Rhinol Laryngol. 2003; 112(3):242-5.

19. L'Estrange P, Bevenius J, Williams L. Intraoral application of microcolpohysteroscopy. A new technique for clinical examination of oral tissues at high magnification. Oral Surg Oral Med Oral Pathol. 1989; 67(3):282-5.

20. Pelucchi S, Bianchini C, Travagli M, Pastore A. Contact endoscopy of the oral mucosa: preliminary results. Acta Otorhinolaryngol Ital. 2007; 27(2):59-61.

21. Ramos GHA, Dedivitis RA, Oliveira BV, Pedruzzi P, França CM. Avaliação das lesões da cavidade oral por endoscopia de contato. Rev Bras Cir Cabeça Pescoço. 2006; 35(2):85-7.

22. Pak MW, To KF, Leung SF, van Hasselt CA. In vivo diagnosis of nasopharyngeal carcinoma using contact rhinoscopy. Laryngoscope. 2001; 111(8):1453-8.

23. Loyola A, Valle E, Lopes LM. In: CPDT- Centro Pré-Natal de Diagnóstico e Tratamento: microcolposcopia. Disponível em h t t p : / / w w w c pdt.com.br/s y s / i n t e r n a . asp?id_secao=3\&id_noticia=51. (Acessado em $05 \mathrm{dez} 2007$ ).

24. Parisi CMA. Microcolposcopia. Roma: UOC Ginecologia Ospedale S. Giovanni. [citado 2007 Dec 5]. Available from: http:/ /www.carmeloparisi.it/microcolposcopia. html

25. Carriero E, Galli J, Fadda G, Di Girolamo S, Ottaviani F, Paludetti C. Preliminary experiences with contact endoscopy of the larynx. Eur Arch Otorhinolaryngol. 2000; 257(2):68-71.

26. WHO Collaborating Center for Oral Precancerous Lesions. Definition of leukoplakia and related lesions: an aid to studies on oral precancer. Oral Surg Oral Med Oral Pathol. 1987; 46(4):518-39.
27. Kolesariæ N, Börner BI, Sader R, Meyer J, Zeilhofer HF. [Early detection and prevention of oral precancer. An enquiry among dentists in Switzerland]. Schweiz Monatsschr Zahnmed. 2007; 117(9):911-9.

28. Tetu E. Recursos diagnósticos: Teste do azul de toluidina. In: Tommasi AF, Garrafa V, editores. Câncer bucal. São Paulo: Medisa. 1980. p.416-21.

29. Gray MGL, Burls A, Elley K. The clinical effectiveness of toluidine blue dye as an adjunct to oral cancer screening in general dental practice. West Midlands DES Report [serial on the Internet]. $2000 \mathrm{Dec}$ [cited $2007 \mathrm{Nov} 20$ ]: [about 57 p.]. Available from: http://www.rep.bham.ac.uk/2000/toludine_blue.pdf

30. Birdsong GG, Davey DD, Darragh TM, Elgert PA, Henry M. Amostra adequada. In: Salomon D, Nayar R, editores. Sistema Bethesda para citopatologia cervicovaginal. Rio de Janeiro: Revinter; 2005. p. 1-11.

Como citar este artigo:

Ramos GH, Tavares MR, Dedivitis RA, França CM, Oliveira BV, Pedruzzi PA. Endoscopia de contato (microestomatoscopia) nas lesões da boca e do lábio: avaliação do método. Rev Col Bras Cir. [periódico na Internet] 2008; 35(6). Disponível em URL: http:// www.scielo.br/rcbc

Endereço para correspondência:

Gyl H. A. Ramos

Rua da Paz 195 sala 12 - Centro

80060160 - Curitiba- PR

Fone: 41- 99482452 e 41-32629322

FAX: 41- 30772465

gharamos@hotmail.com 